Titulo do Trabalho

\title{
INFLUÊNCIA DA TEMPERATURA E PRECIPITAÇÃO NA DISTRIBUIÇÃO DAS ESPÉCIES CRITICAMENTE AMEAÇADAS DE EXTINÇÃO OCORRENTE EM MINAS GERAIS
}

\author{
Nome do Autor (a) Principal
}

Vanessa Leite Rezende

Nome (s) do Coautor (a) (s)

Fernanda Leite Cunha

Instituição ou Empresa

Universidade Federal de Minas Gerais

Universidade Federal de Lavras

E-mail de contato

vanessa.leite.rezende@gmail.com

Palavras-chave

Variáveis ambientais, Flora ameaçada de extinção, distribuição de espécies

\section{INTRODUÇÃO}

A Terra sempre passou por ciclos naturais de aquecimento e resfriamento, assim como nos períodos geológicos foram lançadas quantidades colossais de gases durante milhares de anos, criando um efeito estufa natural. Devido a ação antrópica esse fenômeno tem se intensificado, acarretando em mudanças climáticas que afetam os ecossistemas naturais e especificamente na distribuição de biomas, da biodiversidade, na agricultura e nos recursos hídricos

Marengo 2006 descreve que os gases do efeito estufa retém parte da energia vinda do sol, refletida pela superfície do planeta, e a redistribuem em forma de calor através das circulações atmosféricas e oceânicas. Como uma forma de equilíbrio, 
parte dessa energia é irradiada novamente para o espaço. Qualquer alteração nesse processo afeta o clima global.

Sabe-se que acentuadas mudanças no uso do solo, afetam a maneira como se realiza o ciclo hidrológico no ecossistema (Fonseca Duarte, 2005). Normalmente, em áreas desmatadas, a umidade não evapora e também não fica retida, como acontece na presença da vegetação; com isso as precipitações são afetadas (Laurence, 1998) podendo ocorrer redução de mais de $20 \%$ nos índices pluviométricos.

Em setembro de 2005, houve um aumento de $300 \%$ nas queimadas, em relação ao mesmo período de 2004. Os impactos desses incêndios foram desastrosos tanto para as comunidades afetadas, como clima do planeta, pois a proliferação dos incêndios intensificou as emissões de carbono Marengo 2006. Nepstad et al., (2004) indica que em um estudo do Instituto de Pesquisa Ambiental da Amazônia mostram que, em um quadro de aquecimento global e secas mais frequentes, as florestas da região amazônica perdem muita umidade, tornam-se muito mais vulneráveis às queimadas, a mortalidade de árvores aumenta significativamente e há um aumento nas emissões de carbono para a atmosfera.

Diamond (1989) atribuiu a extinção de espécies por atividades antrópicas ao “Quarteto Diabólico” (“Evil Quartet”): destruição de habitat, sobrematanca, introdução de espécies exóticas e cadeias de extincao.Com os grandes efeitos que as mudanças climáticas tem causado a biodiversidade (Thomas et al., 2004) transformam o "quarteto" de Diamond em "quinteto". Embora os efeitos sinérgicos entre esses cinco elementos desencadeadores possam representar uma ameaça ainda maior as espécies (Brook et al., 2008).

Mittermeier et al., (2005) discutiu que as mudanças do uso da terra levaram a formação de 35 hotspots no mundo, locais com grande biodiversidade de espécies, com altos níveis de endemismo, com grande perda de habitat. No Brasil encontramos dois hotspots: o Cerrado e a Mata Atlântica (Myers, 2000). Além dos altos níveis de endemismo, esses hotspots abrigam diversas espécies ameaçadas de extinção.

As listas vermelhas tornaram-se uma ferramenta essencial para a conservação das espécies (Colyvan et al., 1999) em nível nacional e global. Não apenas por fornecerem dados importantes sobre a atual situação das espécies ameaçadas, (Vie et al., 2009), como também por permitirem o estabelecimento de um vinculo importante entre cientistas e autoridades (Scarano \& Martinelli, 2010). As categorias 
de ameaça das listas vermelhas devem fornecer uma avaliação científica e objetiva da probabilidade de uma espécie se extinguir em dado tempo, caso a circunstância em que a espécie encontra-se permaneça (Mace \& Lande 1991).

Segundo Possingham et al., (2002) as listas vermelhas são importantes ferramentas para avaliar o estado de conservação das espécies de plantas, são geralmente utilizadas para quatro fins: reduzir a exploração de espécies e seus habitats e relatar o estado em que se encontra o meio ambiente; garantir recursos financeiros para a recuperação de espécies; criação de áreas protegidas. Collar, (1996) também inclui utilidades como educação ambiental, geração de novas informações, indicador do progresso de medidas de conservação e como uma oportunidade para compilar dados sobre espécies ameaçada.

\section{OBJETIVO GERAL}

Este trabalho teve o objetivo de levantar as espécies criticamente ameaçadas de extinção em Minas Gerais, e correlaciona-las com variáveis ambientais a fim de verificar os principais fatores ambientais que influenciam na distribuição dessas espécies no estado.

\section{OBJETIVOS ESPECÍFICOS}

Analisar quantas espécies consideradas criticamente ameaçadas de extinção pelo Livro Vermelho (Martinelli \& Moraes, 2013) ocorre no estado de Minas Gerais;

Analisar a distribuição dessas espécies criticamente ameaçadas de Minas Gerais e sua relação com variáveis ambientais;

Verificar as espécies mais correlacionadas com as variáveis ambientais (principalmente com as variáveis de temperatura) a fim de inferir sobre as possíveis consequências das mudanças climáticas na distribuição dessas espécies

\section{METODOLOGIA}


As informações sobre o status de ameaça das espécies foram obtidas do Livro Vermelho da Flora do Brasil (Martinelli \& Moraes, 2013), por ser a lista mais recente publicada para o Brasil. Foram consideradas todas as espécies arbóreas, arbustivas e herbáceas. A partir dessas informações obtivemos os dados de ocorrência dessas espécies a partir do banco de dados o Global Biodiversity Information Facility (http://www.gbif.org/), e SpeciesLink (http://splink.cria.org.br/). Quando a informação sobre as coordenadas geográficas não estava presente nas fontes consultadas utilizou-se as coordenadas dos municípios onde as coletas foram feitas. Esses dados foram conferidos e corrigidos, quando necessário. Nos casos de imprecisão de dados e coordenadas duplicadas, as amostras foram retiradas da análise. Os dados foram plotados no software DIVA-GIS (Hijmans et al. 2011). Os dados das variáveis climáticas e de relevo dos hábitats conhecidos da espécie foram extraídos do banco de dados "World-Clim" (http://www.worldclim.org; Hijmans et al., 2005), através da ferramenta "Extract value by points" disponíveis no DIVA-GIS. Foram obtidas 18 variáveis bioclimáticas e altitude com extensão de $5 \mathrm{~km}$. A matriz final foi composta por nove espécies e 36 áreas amostrais.

Inicialmente foi feita uma análise de componentes principais (PCA) sobre a matriz de variáveis bioclimáticas. A PCA é uma técnica de ordenação bastante aplicada em ecologia (Valentin, 1995) e é utilizada pela sua capacidade de compressão dos dados em função da existência de correlação entre diversas variáveis medidas, uma vez que concentra a maior parte da informação em poucas variáveis, diminuindo assim a dimensionalidade dos dados, sem perda significativa da informação (Sabin et al., 2004). No presente trabalho, a PCA foi usada com o objetivo de detectar e remover redundâncias entre as variáveis bioclimáticas, indicadas por sobreposição direta ou inversa dos autovetores. Em cada grupo de variáveis colineares, apenas a mais importante para os padrões florísticos foi mantida. A importância de cada variável para os padrões florísticos foi checada mediante uma CCA (análise de correspondência canônica) preliminar, usando o programa Fitopac (Shepherd, 2010). A CCA tem como objetivo ordenar unidades amostrais em função de um determinado grupo de variáveis ambientais (Coimbra et al., 2007). Essa análise é frequentemente utilizada para identificar gradientes ambientais em bancos de dados específicos, particularmente quando as variáveis ambientais são importantes a fim de se determinar a composição da comunidade (ter Braak, 1987). 
Foram preparadas duas matrizes para a CCA final: principal (que consistia na presença e ausência das espécies nas áreas) e secundária (variáveis ambientais nas mesmas áreas). Da matriz principal, foram excluídas espécies de baixa ocorrência, ou seja, aquelas que apareceram apenas uma vez em todas as áreas analisadas. Após esse procedimento, essa matriz ficou constituída por $\mathrm{x}$ áreas $\mathrm{e} x$ espécies. As variáveis ambientais presentes na matriz secundária foram Altitude, Bio 17 (precipitação no trimestre mais seco), Bio 15 (sazonalidade da precipitação), Bio 18 (precipitação no trimestre mais quente), Bio 3 (isotermalidade), Bio 8 (temperatura média no trimestre mais úmido) e Bio 9 (temperatura média no trimestre mais seco). A CCA foi processada com 999 permutações de Monte Carlo, para testar o nível de significância dos eixos de ordenação canônica.

\section{RESULTADO (S)}

Foram encontradas nove espécies criticamente ameaçadas com dados no Species Link (http://splink.cria.org.br/), para o estado de Minas Gerais. Dentre essas espécies cinco (Mimosa thomista, Agalinis schwackeana, Canastra lanceolata, Comanthera brasiliana, Dichorisandra glaziovi) apresentam hábito herbáceo e/ou arbustivo, duas (Aspilia diniz, Auspilia Almasensis) apresentam hábito exclusivamente arbustivo. De hábito arbóreo e liana foram encontradas apenas uma espécie de cada (Araucaria angustifolia e Stigmaphyllon macedoanum) respectivamente (CNCflora, 2012).

Com base nos resultados da CCA pode-se constatar que as variáveis mais correlacionadas com o eixo 1 foram Bio $3(-0.927)$ e Bio $9(0,795)$, e com o segundo eixo foram Bio $15(-0,650)$ e Bio $17(0,554)$. Assim, podemos observar que as variáveis relacionadas a temperatura e a altitude foram mais correlacionadas com o eixo 1, e as variáveis relacionadas a precipitação apresentaram correlações semelhantes para os dois eixos. Podemos notar também, para o primeiro eixo da ordenação, que as espécies se dividiram formando dois grupos; espécies mais correlacionadas com as variáveis de precipitação e altitude, e espécies mais correlacionadas com as variáveis de temperatura.

As espécies mais correlacionadas com as variáveis de precipitação e altitude foram M. thomista, A. angustifolia, A. schwackeana e A. diniz, espécies restritas e 
endemicas de regiões montanas de Minas Gerais (Martinelli \& Moraes, 2013). A espécie M. thomista, ocorre nos Campos Rupestres em altitudes que variam entre entre $1200 \mathrm{~m}$ e $1300 \mathrm{~m}$. A. schwackeana é endêmica da Serra do Cipó e, a espécie $A$. diniz é endêmica da Serra do Caraça. A espécie $A$. angustifolia está relacionada a altas altitudes na região sudeste do Brasil devido as diferentes condições climáticas encontradas nas regiões montanhosas em relação as áreas mais baixas do sudeste, porém na porção subtropical do Brasil, pode ser encontrada em altitudes inferiores devido as menores temperaturas e precipitação constante encontradas nessa região (Backs, 2009).

Já as espécies C. lanceolata, C. brasiliana, A. Almasensis, D. glaziovi, $S$. macedoanum, foram mais correlacionadas com as variáveis de temperatura. Dentre essas espécies, C. lanceolata é endêmica da Serra da Canastra, C. brasiliana é restrita à região da Pedra Menina, ocorrendo em vegetação rupestre de solo arenoso e em altitudes inferiores a 1000m. A. Almasensis é endêmica do Cerrado e dos Campos Rupestres (700 e 1600m de altitude) ocorrendo também no estado da Bahia. D. glaziovii é possui distribuição restrita aos campos rupestres da Serra do Espinhaço, ocorrendo entre 750m e 1000m altitude. E S. macedoanum, ocorre no bioma Cerrado, exclusivamente no estado de Minas Gerais.

Foi possível notar que as espécies de hábito arbustivo e/ou arbóreo foram mais correlacionadas com as variáveis de precipitação e altitude, enquanto as espécies que apresentam hábito herbáceo e herbáceo/arbustivo foram mais correlacionadas com as variáveis de temperatura.

\section{CONSIDERAÇÕES FINAIS}

Nossos resultados mostraram associações significativas das variáveis ambientais com a flora criticamente ameaçada de Minas Gerais, o que sugere que essas espécies são altamente influenciadas pelas condições climáticas existentes no ambiente em que vivem. Assim a preservação, principalmente, das regiões montanhosas do estado é essencial para manter os processos ecológicos e de evolução biológica dessas espécies, o que pode ser particularmente importante no presente cenário de mudanças climáticas. Além disso, o uso da terra em larga escala 
e as mudanças da cobertura que estão ocorrendo rapidamente nos trópicos (Mayaux et al., 2005), conduzem à extrema fragmentação de cobertura vegetal, efeitos de bordas, e redução da área dos fragmentos nativos (Fischer \& Lindenmayer, 2007). Em Minas Gerais é notável uma forte pressão exercida principalmente pela mineração, agricultura e especulação imobiliária. A consideração de que a distribuição das espécies criticamente ameaçadas pode ser determinada por fatores climáticos, têm importantes implicações para o conhecimento e conservação da flora ameaçada e para a nossa capacidade de mantê-las no futuro.

\section{REFERÊNCIAS}

Backs Albano. Distribuição atual da Floresta com Araucária: condicionamento climático. In: Fonseca CR, Souza AF, Leal-Zanchet AN, Dutra T, Backs A, Ganaddo G (eds) Floresta com Araucária: ecologia, conservação e desenvolvimento sustentável. Holos, Ribeirão Preto, pp 39-44. 2009.

Brook, Barry; Sodhi, Navjot; Bradshaw, Corey. Synergies among extinction drivers under global change. Trends Ecol. Evol. 23, 453-460. 2008.

CNCFlora - Centro Nacional de Conservação da Flora (2012). http://cncflora.jbrj.gov.br/. Accessed 20 Sept. 2014.

Coimbra, Rogério Anadrade; TOMAZ, Camila Aquino; MARTINS, Cibele Chalita; NAKAGAWA, João. Teste de germinação com acondicionamento dos rolos de papel em sacos plásticos. Revista Brasileira de Sementes, v.29, n.1, p.92-97, 2007.

Collar, N.J. 1996. The Reasons for Red Data Books. Oryx 30:121-130.

Colyvan, Mark; Burgman, Mark; Todd, Charles; Akçakaya, Resit; Boek, Chris. The treatment of uncertainty and the structure of IUCN threatened species categories.

Biological Conservation. (89) 245- 249. 1999.

Diamond, Jared. 1989. Overview of Recent Extinctions. In: Western, D., Pearl, M.C., (eds.). Conservation for the Twenty-First Century. Oxford: Oxford University Press, p. 37-41.

Fischer, Joern; Lindenmayer, David. Landscape modification and habitat fragmentation: a synthesis. Global Ecology and Biogeography. (16). 265-280. 2007.

Fonseca Duarte, Alejandro. Variabilidade e tendência das chuvas em Rio Branco, Acre, Brasil. Revista Brasileira de Meteorologia, v.20, n.1, 37-42, 2005.

Hijmans, Robert J.; Cameron, Susan. E.; Parra, Juan. L.; Jones, Peter. G.; Jarvis, Aandy. Very high resolution interpolated climate surfaces for global land areas. International Journal of Climatology 25, 1965-1978. 2005.

Hijmans, Robert J; Guarino, Luigi; Bussink, Coen.; Mathur, Prem; Cruz, Mariana; Barrentes, Israel; Rojas, Edwin. DIVA- GIS.v7.4. A geographic information system for the analysis of species distribuition data. 2011. Disponível em: /www.diva-gis.org. Acesso em 10 de jun. 2014. 
Laurance, William. A crisis in the making: responses of Amazonian forests to land use and climate change.Trends in Ecology and Evolution. (13) 411-415, 1998.

Mace, G.M. \& Lande, R. 1991. Assessing Extinction Threats: Towards a Re-evaluation of IUCN Threatened Species Categories. Conservation Biology 5:148-157

Martinelli Gustavo; Moraes Miguel Avila. Livro vermelho da flora do Brasil. Jardim Botânico do Rio de Janeiro, Rio de Janeiro. 2013.

Marengo, José. Mudanças climáticas globais e seus efeitos sobre a biodiversidade: caracterização do clima atual e definição das alterações climáticas para o território brasileiro ao longo do século XXI. Brasília: MMA, 2006.

Mayaux Philippe; Holmgren Peter; Achard Fréderic; Eva Hugh; Stibig Hans-Jurgen; Branthomme Anne. Tropical forest cover change in the 1990's and options for future monitoring. Philosophical Transactions of The Royal Society B: Biological Sciences, 360, 373-384. 2005.

Myers, Norman; Mittermeier, Russell; Mittermeier, Cristina, Fonseca, Gustavo; Kent, Jennifer. Biodiversity hotspots for conservation priorities. Nature 403: 853-858. 2000.

Mittermeier, Russel; Fonseca, Gustavo; Rylands, Anthony; Brandon Katrina. A brief history of biodiversity conservation in Brazil. Conservation Biology 19(3): 601-611. 2005.

Nepstad, Daniel; Lefebvre, Paulo; Lopes da Silva, Urbano; et al. Amazon drought and its implications for forest flammability and tree growth: a basin-wide analysis. Global Change Biology (10) 704-717. 2004.

Possingham, H.P., Andelman, S.J., Burgman, M.A., Mendellíns, R., Master, L.L. \& Keith, D. 2002. Limits to the Use of Threatened Species List. Trends in Ecology and Evolution 17:503-507.

Sabin, Janusa Goelazer; Ferrão, Marco Floreas; Furtado, João Carlos. Análise multivariada aplicada na identificação de fármacos antidepressivos. Parte II: Análise por componentes principais (PCA) e o método de classificação SIMCA. Revista Brasileira de Ciências Farmacêuticas, São Paulo, v. 40, n. 3, p.387-395, 2004.

Scarano, F.R. \& Martinelli, G. 2010. Brazilian List of Threatened Plant Species: Reconciling Scientific Uncertainty and Political Decision-making. Natureza \& Conservação 8:13-18.

Shepherd, George .J. Fitopac 2.1.2.85. Manual do Usuário. Departamento de Botânica, Universidade Estadual de Campinas, Campinas, SP. 2010.

ter Braak, Cajo. J. F. The analysis of vegetation environment relationship by canonical correspondence analysis. Vegetatio 69:69-77. 1987.

Thomas, Chris; Cameron, Alison; Green, Rhys et al. Extinction risk from climate change. Nature. (427) 145-148. 2004.

Valentin, Jean Louis. Agrupamento e ordenação. In Tópicos em tratamentos de dados biológicos (P.R. Peres-Neto, J.L. Valentin \& F.A.S. Fernandez, eds.). Oecologia Brasiliensis, v. II, PPGE-UFRJ, Rio de Janeiro, p. 25-55. 1995.

Vié, Jean-Cristopher.; Hilton-Taylor, Craick; Stuart, Simon. Wildlife in a Changing World - An Analysis of the 2008 IUCN Red List of Threatened Species. Gland, Switzerland: IUCN. 180 p. 2009. 